\title{
PRECISE DETERMINATION OF EARTH'S CENTER OF MASS USING MEASUREMENTS FROM THE GLOBAL POSITIONING SYSTEM
}

\author{
Yvonne Vigue, Stephen M. Lichten, Geoffrey Blewitt, \\ Michael B. Heflin, and Rajendra P. Malla \\ Jet Propulsion Laboratory, California Institute of Technology
}

\begin{abstract}
Global Positioning System (GPS) data from a worldwide geodetic experiment were collected during a 3 week period early in 1991 . We estimated geocentric station coordinates using the GPS data, thus defining a dynamically determined reference frame origin which should coincide with the Earth center of mass, or geocenter. The 3-week GPS average geocenter estimates agree to $7-13 \mathrm{~cm}$ with geocenter estimates determined from satellite laser ranging, a well-established technique. The RMS of daily GPS geocenter estimates were $4 \mathrm{~cm}$ for $x$ and $y$, and $30 \mathrm{~cm}$ for $z$.
\end{abstract}

Introduction

The satellites of the Global Positioning System (GPS) can be used to precisely measure global geodynamical quantities such as changes in Earth's rotation vector and rotation rate, crustal motion, and location of the Earth's center of mass. A recent opportunity to test these capabilities was provided by the 1991 GPS IERS (International Earth Rotation Service) and Geodynamics experiment (GIG), which took place Jan 22 - Feb 13 1991. Although only 15 GPS satellites were operational, the GPS constellation will include 24 satellites when complete and substantial improvement in the accuracy of geodetic measurements is expected in the next few years. The Earth orientation and baseline results recently published (Herring et al., 1991, Heflin et al, 1992, Lichten et al., 1992, Lindqwister et al., 1992) show that even such limited GPS data provided centimeter-level accuracy for measurements of Earth orientation variations and ground station baselines.

The location of the Earth's center of mass, or geocenter, is a fundamental measurement which has geophysical and practical significance. The definition of a terrestrial reference frame requires specification of the origin, scale, and orientation of a coordinate system. Since the mass distribution of the Earth is constantly changing, it is important to measure the time variations of the geocenter relative to points on the Earth's crust. Since geodetic measurements are often made with different techniques and instruments in slightly different terrestrial reference frames, precise measurement of the origin, scale, and orientation of these frames relative to one another is needed in order to combine different geodetic data. Geocenter estimates presented in here are based on a comparison with the SV5 reference frame [Murray et al., 1990] origin. The SV5 frame is constructed from both satellite laser

Copyright 1992 by the American Geophysical Union.

Paper number 92GL01575

0094-8534/92/92GL-01575\$03.00 ranging (SLR) and very long baseline interferometry (VLBI) data. The SV5 scale and origin are from SLR, and the origin is chosen to be the geocenter.

Since GPS satellites move in high-Earth orbits, they are relatively insensitive to errors in the gravity field, particularly for relatively short data ares of a few days or less [Bertiger et al., 1986]. With each ground site tracking 5-6 GPS satellites simultaneously, the geocenter can be estimated quickly (in a few days or less). Use of GPS with short arcs to quickly determine the geocenter would in principle enable time variations over a few days or less to be measured for the first time, without complications of re-estimation of the gravity field. In this paper, we present the first decimeter-quality GPS solutions for the geocenter which we obtained from 21 days of data. We discuss not only accuracy by comparing with other independent data, but also short-term (daily) precision of the estimates and the potential for further improvement.

Over 100 globally distributed tracking sites participated in the GIG' 91 experiment. Only data from the 21 Rogue GPS receiver sites (Figure 1 and Table 1) were used in our analysis. The Rogue receiver is described by Srinivasan et al. [1989]. The sites cover $+79^{\circ}$ to $-35^{\circ}$ latitude, but only 4 of these 21 sites were located in the southern hemisphere. Data from Jan 31 and Feb 13 were not included in this analysis since an input error in antenna height was discovered. Data from satellite PRN6 was not used because it was spinning.

\section{Estimation Strategy}

The data collected during the GIG'91 experiment have been processed using the GIPSY (GPS Inferred Positioning SYstem) software [Lichten and Border, 1987; Sovers and Border, 1990]. A standard estimation technique is used in this analysis, consisting of single-day arc solutions. Three sites are fixed as fiducial sites in the SV5 reference frame, while all other station locations are estimated. A fiducial network can establish a well-defined terrestrial reference frame with 2 or 3 receivers fixed to their known coordinates [Malla and $W u, 1989]$. The fixed vectors between fiducial sites define orientation and scale in accordance with SV5 [Murray et al., 1990]. The fiducials adopted for this study are Troms $ø$, Norway; Pinyon, California (USA); and Wettzell, Germany. Satellite orbits, non-fiducial station locations, and 3 components of the geocenter offset from the nominal origin are estimated. The satellite orbits are estimated about a dynamically defined origin which determines the Earth's center of mass, or geocenter. The 3 estimated geocenter parameters represent a translational offset between the origin of the reference frame and the dynamical origin defined by the 


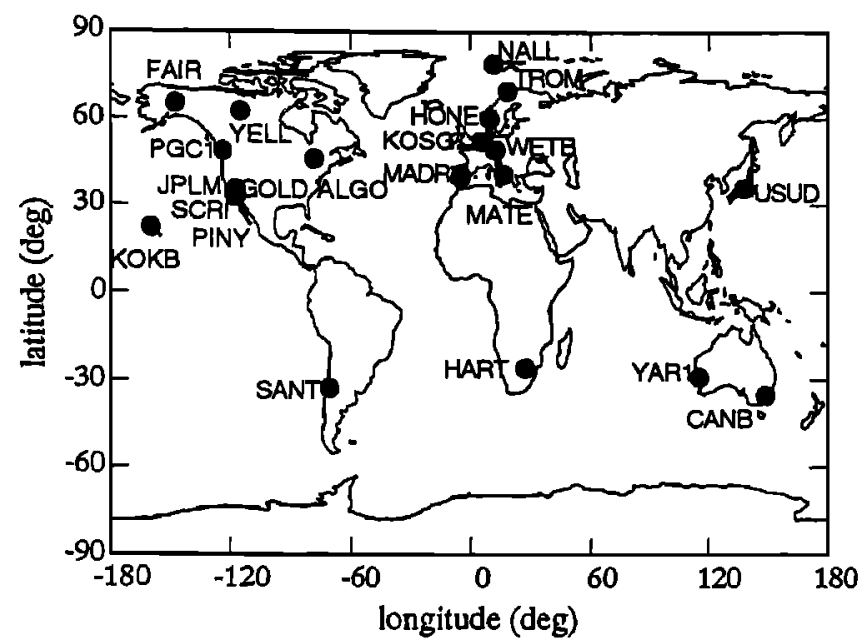

Fig. 1: GIG'91 GPS Rogue Receiver Locations

GPS orbits. Thus geocentric station locations are determined for all non-fiducial sites. Earth orientation parameters, 3 solar radiation coefficients per satellite, GPS carrier phase biases, random walk zenith troposphere delays for each site, and transmitter/receiver clocks were also estimated. Details on the estimation strategy are given by Lichten [1990a, 1990b].

An alternative no-fiducial technique demonstrated by Heflin et al. [1992] involves estimating all station coordinates and satellite positions with very weak constraints $(\sim 1 \mathrm{~km})$. This defines a rigid network which has an ill-conditioned orientation but is nevertheless constrained by the data to the geocenter through the dynamics of the GPS satellites. To define geocentric coordinates, the network can be translated, rotated, and scaled into the reference frame of choice (e.g., SV5). The 3 estimated translational parameters are the geocenter offset from the origin of this frame [Blewitt et al., 1992].

\section{Results and Discussion}

Figure 2 shows daily $x$ and $y$ geocenter component estimates as offsets from the nominal values. The nominal values used for each component of the geocenter come from the global SV5 station location solutions. The $x$ and $y$ components of the geocenter exhibit variations which appear to be negatively correlated. An error analysis predicts this covariant behavior, and it is thought to be due to the asymmetric global distribution of sites. The weighted mean values (3-week weighted GPS averages) are $8.3 \mathrm{~cm}$ and $13.4 \mathrm{~cm}$,

\section{TABLE 1: GIG'91 GPS Rogue Receiver Sites}

\begin{tabular}{ll}
\hline ALGO-Algonquin, Canada & NALL-Ny Alesund, Norway \\
CANB-Canberra, Australia & PINY-Pinyon, Califomia(USA) \\
FAIR-Fairbanks, Alaska (USA) & PGC1Victoria, Canada \\
GOLD-Goldstone, Califomia (USA) & SANT-Santiago, Chile \\
HART-Hartebeesthoek, South Africa & SCRI-La Jolla, California (USA) \\
HONE-Honefoss, Norway & TROM-Tromso, Norway \\
JPLM-Pasadena, California (USA) & WETB-Wettzell, Germany \\
KOKB-Kokee, Hawaij (USA) & USUD-Usuda, Japan \\
KOSG-Kootwijk, Netherlands & YAR1-Yarragadee, Australia \\
MATE-Matera, Italy & YELL-Yellowknife, Canada \\
MADR-Madrid, Spain &
\end{tabular}

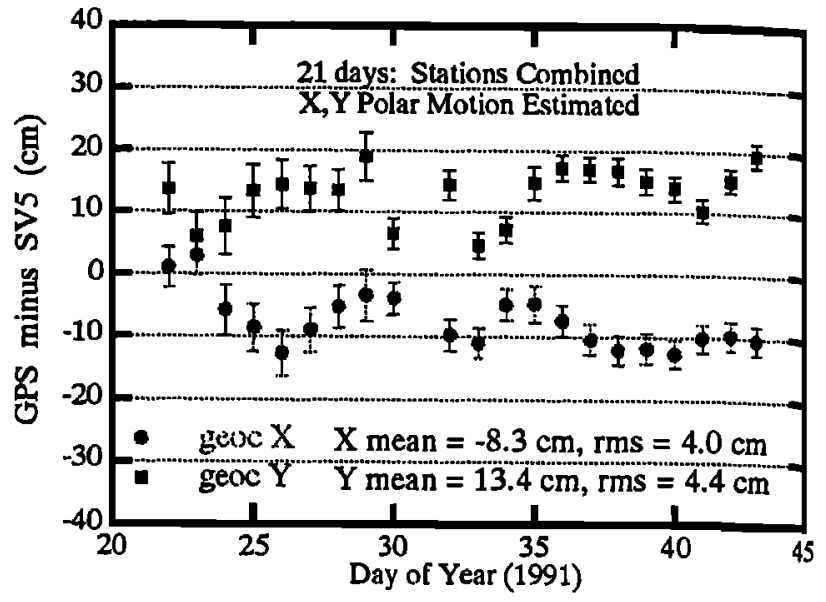

Fig. 2: GPS Geocenter Estimates - $x, y$ comp.

for $\mathrm{x}$ and $\mathrm{y}$ components, respectively; these values represent the difference between the GPS solution for the geocenter and the nominal values, which are based on years of SLR data and are believed to be accurate to better than $5 \mathrm{~cm}$ [Eanes et $a l ., 1990]$. The daily ms scatter about the mean for the $\mathrm{x}$ and $y$ components of the geocenter are $4.0 \mathrm{~cm}$ and $4.4 \mathrm{~cm}$, respectively. This daily rms scatter is a measure of irternal precision of the technique presented here, and can be considered an indication of the potential to measure variations in the geocenter over one day.

The estimates for the geocenter $\mathrm{z}$ component from 3 weeks of GIG'91 data are shown in Figure 3. This component shows larger variations than the $x$ and $y$ components, but we do not believe that these results show actual daily center of mass variations. The 3-week weighted mean offset from nominal for the $z$ component is $7.7 \mathrm{~cm}$, while the daily $\mathrm{ms}$ scatter is $30 \mathrm{~cm}$. It is likely that the estimation of this component is much more sensitive to the uneven distribution of fiducial and non-fiducial stations between the northern and southern hemispheres, and to the uneven 1991 GPS constellation. Although the $z$ component formal errors are are indeed larger than those for the $x$ and $y$ components, the daily $\mathbf{z}$ geocenter formal errors are about one-half as large as the observed rms scatter. Additional systematic error

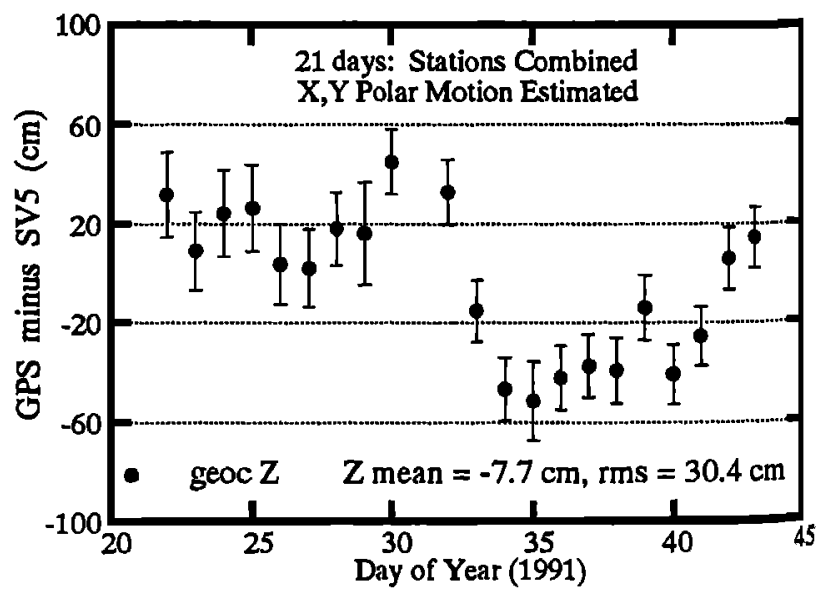

Fig. 3: GPS Geocenter Estimate - $z$ component 
contributions for the $z$ component (as compared to $x$ and $y$ ) may be due to the weak coverage in the southern hemisphere during the experiment. For instance, Hartebeesthoek, a southern hemisphere site whose data were geometrically important, was operating less than 12 hours per day during this experiment. Simulations show that the sigmas for $x, y$, and $z$ geocenter estimates are nearly the same when using a full 24-satellite constellation and full ground coverage between the northern and southern hemispheres [Malla et al., 1992]. The $z$ component also appears to exhibit a discontinuity near February 1 , which is not well understood. Many alternative analysis techniques were tested in an attempt to determine what systematic errors may have caused the discrepancy in the $z$ geocenter estimates between the first and second half of the experiment. Omitting a questionable station or satellite did not change this pattern.

Variations in the estimation strategy were studied in an effort to observe changes in the geocenter estimates. Different fiducial networks yielded little variation in the results, although the different networks we tried did not result in a wide variation of the fiducial network geometry. A sensitivity analysis showed that a $3 \mathrm{~cm}$ fiducial error in all components $(x, y, z)$ would give an error of approximately $9-15 \mathrm{~cm}$ in the geocenter, although this error would be expected primarily as an overall bias with smaller day-to-day variations. The $3 \mathrm{~cm}$ fiducial error" refers to independent 3-cm errors in each of $x$, $y, z$ components for each of 3 fiducials, with no error cancellation assumed. However, results of Blewitt et al. [1982] inidicate that fiducial errors are more likely to be at the level of $1-2 \mathrm{~cm}$. Both the fiducial and non-fiducial sites were not well-distributed in $z$ during GIG'91, thus magnifying errors in the z-component. With a full constellation of 24 GPS and equal north-south hemisphere coverage, the ratio of fiducial to geocenter error is closer to 1:1, according Malla and $W u$ [1989].

Polar motion (Earth orientation) parameters were held fixed in another case, with only slight degradation in the geocenter estimates. We also estimated Earth rotation (UT1UTC) stochastically (every 12 hours) producing little change.

Longer orbit arc lengths were also studied. In these cases, eclipsing satellites' orbits were estimated as single-day arcs to minimize orbit modeling error. Eclipsing satellites are satellites whose orbit orientation is such that the satellites enter a shadowed region where the sun's radiation effects on the spacecraft are reduced (penumbra) or completely blocked (umbra) by the Earth. In general, eclipsing satellite orbit errors have been found to be significantly larger than those for

TABLE 2: Comparison of Geocenter Estimates Between Fiducial and Seven Parameter Transformation Techniques

\begin{tabular}{lc}
\hline $\begin{array}{c}\text { GPS estimates of the } \\
\text { geocenter offset using fiducials }\end{array}$ & $\begin{array}{c}\text { GPS estimates of the } \\
\text { geocenter offset by estimation } \\
\text { of a 7 Parameter Transformation }\end{array}$ \\
\hline$G x=-8.3 \mathrm{~cm}+/-2.7 \mathrm{~cm}$ & $\mathrm{Tx}=-7.5 \mathrm{~cm}+/-2.6 \mathrm{~cm}$ \\
$\mathrm{~Gy}=13.4 \mathrm{~cm}+/-2.4 \mathrm{~cm}$ & $\mathrm{Ty}=13.0 \mathrm{~cm}+/-2.5 \mathrm{~cm}$ \\
$\mathrm{Gz}=-7.7 \mathrm{~cm}+/-13.7 \mathrm{~cm}$ & $\mathrm{Tz}=-14.8 \mathrm{~cm}+/-13.8 \mathrm{~cm}$ \\
\hline
\end{tabular}

non-eclipsing satellites [Schutz et al., 1990]. The 2-day arc case yielded mixed results. One fiducial network slightly reduced the variations and another fiducial network slightly increased them. For 3-day arcs, the results degraded further.

Table 2 shows a summary of the geocenter estimates obtained using the technique presented in this paper as compared with those in Blewitt et al. [1992] where a 7-parameter transformation ( 3 translations, 3 rotations, and a scale factor) is estimated. Tx, Ty, and Tz shown in Table 2 are the 3 translational offsets of the geocenter from the reference frame origin; the values from each technique agree very well in a statistical sense. The differences are much less than the corresponding 1- $\sigma$ formal uncertainties of the estimates. Both techniques use GIG'91 data but use slightly different strategies in obtaining the geocenter offsets. It is important to note that Blewitt et al. [1992] uses 12 sites collocated with the International Terrestrial Reference Frame (ITRF) [IERS, 1991]. Since SV5 is intended to be aligned with ITRF, it is not surprising that the results are in close agreement.

\section{Gravity Field Sensitivity Analysis}

The use of GPS to determine the geocenter from the dynamical information in the orbits may be a powerful technique since the large number of GPS satellites enables full geographical coverage of the globe with a relatively small number of ground stations (10-20) in a day or less. This enables rapid determination of the geocenter with GPS data. In addition, the relatively high altitude of GPS satellites (more than twice the distance from the center of the Earth as LAGEOS) makes the GPS solutions much less sensitive to drag and gravity mismodeling. To quantify this, we examined the effect of gravity errors on the GPS geocenter estimates.

Our analysis computes the error which is introduced by estimating the geocenter with GPS data while leaving the gravity field unadjusted. The effect of not estimating certa in parameters can be quantified through a sensitivity, or consider analysis. In this type of error analysis, the filter computes the sensitivity the estimated parameters to those parameters not being estimated. Let vector $y_{c}$ contain the considered (unestimated) parameters, and $\widehat{\mathbf{X}}, \widehat{\mathbf{P}}$ are the computed filter estimates and covariance (not including effects from the considered parameters). Then the sensitivity matrix, $S$ is defined as

$$
\mathrm{S}=\frac{\partial(\mathrm{X}-\widehat{\mathbf{X}})}{\partial \mathrm{y}_{c}}
$$

where $S$ is calculated from the measurement partials (see Bierman [1977]). The total error covariance, including the formal errors computed in the filter as well as the error contribution from unestimated (considered) parameters, is

$$
\mathrm{P}_{c o n}=\widehat{\mathrm{P}}+\mathrm{SP}_{c} \mathrm{~S}^{\mathrm{T}}
$$

where $\mathrm{P}_{c}$ is the a priori covariance for the consider parameters. Usually, $P_{c}$ is a diagonal matrix, but a more realistic representation sometimes utilizes a full matrix in order to account for correlations between considered parameters. For our analysis, the consider parameters are simply the gravity coef- 
ficients. A very simple and somewhat abbreviated analysis by Bertiger et al. [1986] indicated that the effect of gravity errors on GPS orbits determined from less than 24 hours of data was an order of magnitude smaller than other errors such as data noise, fiducial errors, etc. One would infer from this that other GPS-inferred quantities, such as the geocenter, would also be relatively unaffected by gravity field errors. However we re-analyzed this using the full GEM-T2 covariance [Marsh et al., 1990] for $\mathbf{P}_{\boldsymbol{c}}$ with the global tracking network for GIG'91, in order to better understand the effect of leaving the gravity field fixed while estimating a new origin (geocenter). The new calculations show that the errors introduced in the GPS geocenter estimates from holding the gravity field fixed are only $0.05 \mathrm{~mm}, 0.7 \mathrm{~mm}$, and $0.5 \mathrm{~mm}$ in the $x, y$, and $z$ components. These errors are less than $1 \%$ of other observed errors and are clearly insignificant in the overall GPS geocenter error budget.

\section{Summary and Conclusions}

The mean GPS geocenter estimate from a 3-week 1991 experiment agrees to $7-13 \mathrm{~cm}$ in each component when compared with an SLR (SV5) reference measurement. Individual single-day GPS solutions exhibited rms variations of $4 \mathrm{~cm}$ in $x$ and $y$ and $30 \mathrm{~cm}$ in $z$. Geocenter estimates made with a standard fiducial strategy and with a fiducial-free 7 parameter transformation produce similar answers, with formal errors of $2-3 \mathrm{~cm}$ in $x$ and $y$, and $15 \mathrm{~cm}$ in $z$. The incomplete GPS constellation and very limited southern hemisphere ground data are believed to be factors limiting the precision and accuracy of daily estimates from the 1991 data set. With satellite launches in the near future, the GPS constellation will grow by about $50 \%$. Additional southern hemisphere data should improve the $z$ component of the geocenter particularly. GPS geocenter accuracy on a daily basis with a full constellation and uniform global network is expected to be better than $5 \mathrm{~cm}$; incorporation of data from low earth orbiters such as TOPEX is expected to strengthen the solution even more [Malla et al., 1992]. Further GPS and SLR intercomparisons could potentially uncover systematic errors that may exist in either technique.

Acknowledgements. The work described in this paper was carried out by the Jet Propulsion Laboratory, California Institute of Technology, under contract with the National Aeronautics and Space Administration. We thank the many participants who helped make GIG'91 a success, and Yoaz Bar-Sever and Ron Muellerschoen, who contributed to special software used in this work.

\section{References}

Bertiger, W., S. Wu, J. Border, S. Lichten, B. Williams, and J. Wu, High Precision GPS Orbit Determination Using March 1985 Demonstration Data, AIAA 24th Aerospace Sciences Meeting, paper ALAA-86-0089, 1986.

Bierman, G., Factorization Methods for Discrete Sequential Estimation, V128, Academic Press, New York, 1977.

Blewitt, G., M. Heflin, F. Webb, U. Lindqwister and R. Malla, Global Coordinates with Centimeter Accuracy in the
International Terrestrial Reference Frame Using GPS, Geophys. Res. Lett., 19, No. 9, 853-856, 1992.

Eanes, R., B. Schutz, B. Tapley and M. Watkins, Earth Orientation Results from CSR 89L02, IERS Technical Note 5, p.51, Observatoire de Paris, 1990.

Heflin, M., W. Bertiger, G. Blewitt, A. Freedman, K. Hurst, S. Lichten, U. Lindqwister, Y. Vigue, F. Webb, T. Yunck, and J. Zumberge, Global Geodesy Using GPS Without Fiducial Sites, Geophys. Res. Lett., 19, 131-134, 1992.

Herring, T., D. Dong, and R. King, Sub-milliarcsecond Determination of Pole Position Using Global Positioning System Data, Geophys. Res. Lett., 18, pp. 1893-1896, 1991.

IERS, Annual Report for 1990, IERS Central Bureau, Observatoire de Paris, 1991.

Lichten, S., S. Marcus, and J. Dickey, Sub-Daily Resolution of Earth Rotation Variations with Global Positioning System Measurements, Geophys. Res. Lett., 19, $537-$ 540, 1992.

Lichten, S., Towards GPS Orbit Accuracy of Tens of Centimeters, Geophys. Res. Lett., 17, 215-218, 1990a.

Lichten, S., Estimation and Filtering for High-Precision GPS Positioning Applications, Manus. Geod., 15, 159-176, 1990b.

Lichten, S., and J. Border, Strategies for High Precision Global Positioning System Orbit Determination, $J$. Geophys. Res., 92, 12751-12762, 1987.

Lindqwister, U., A. Freedman, and G. Blewitt, Daily Estimates of the Earth's Pole Position with the Global Positioning System, Geophys. Res. Lett., 19, 845-848, 1992.

Malla, R., and S. Wu, GPS Inferred Geocentric Reference Frame for Satellite Positioning and Navigation, Bull. Geod., 63, 263-279, 1989.

Malla, R., S. Wu, and S. Lichten, Geodetic Reference Frame for Geodynamics, J. Geophys. Res., Solid Earth: Modelling Crustal Deformation Special Section, In Press, 1992.

Marsh, J., F. Lerch, B. Putney, T. Felsentreger, B. Sanchez, S. Klosko, G. Patel, J. Robbins, R. Williamson, T. Engelis, W. Eddy, N. Chandler, D. Chinn, S. Kapoor, K. Rachlin, L. Braatz, and E. Pavlis, The GEM-T2 Gravitational Model, J. Geophys. Res., 95 (B13), 22043-22071, 1990.

Murray, M., R. King, and P. Morgan, SV5: A Terrestrial Reference Frame for Monitoring Crustal Deformation with the Global Positioning System, (Abstract), EOS Trans, AGU, 71, p. 1274, 1990.

Schutz, B., C. Ho, P. Abusali, and B. Tapley, CASA UNO GPS Orbit and Baseline Experiments, Geophys. Res. Lett., 17, 643-646, 1990.

Sovers, O., and J. Border, Observation Model and Parameter Partials for the JPL Geodetic GPS Modelling Software "GPSOMC", JPL Pub. 87-21, Rev. 2, Jet Propulsion Laboratory, Pasadena, CA, 1990.

Srinivasan, J., T. Meehan, and L. Young, Code and Codeless Ionospheric Measurements with NASA's Rogue GPS Receiver, Proceedings Inst. of Navigation GPS-89 Conference, 1989, 451-454 (Inst. of Navigation).

Geoff Blewitt, Michael Heflin, Steve Lichten, Rajju Malla, and Yvonne Vigue, Jet Propulsion Laboratory, Mail Stop 238-600, 4800 Oak Grove Drive, Pasadena, CA 91109.

(Received March 10, 1992; accepted May 8, 1992.) 